\title{
LANÚS, EXPERIENCIAS FORMATIVAS INTERDISCIPLINARIAS: ANTECEDENTES DE LA SALUD MENTAL COMUNITARIA
}

\author{
María Marcela Bottinelli \\ Mariela Nabergoi \\ Daniel Frankel \\ Sergio Esteban Remesar \\ Francisco Manuel Diaz \\ Andrea Fabiana Albino \\ Carolina Ruth Maldonado \\ Ana Cecilia Garzon \\ Universidad Nacional de Lanús, Argentina
}

\begin{abstract}
RESUMEN
Este artículo caracteriza los supuestos epistemológicos en las propuestas formativas del Servicio de

Psicopatología del Hospital Aráoz Alfaro (“El Lanús”) y los posgrados en Salud Mental Comunitaria de la Universidad Nacional de Lanús (SMC UNLa). Con metodologías cualitativas y triangulación de fuentes, desde un paradigma comprensivista realizamos entrevistas a 6 referentes, 74 encuestas a estudiantes, egresadxs y graduadxs de posgrado; y relevamos 92 documentos. Los resultados destacan las implicancias del Lanús en espacios de gestión, diseño de políticas nacionales y formación del Área de SMC UNLa. Ambas experiencias con sus singularidades comparten una misma base epistemológica e incluyen aspectos formativos que permiten visibilizar y potenciar las construcciones históricas alternativas en el campo de salud mental así como replicarlas desde la experiencia interdisciplinaria y participativa en y con la comunidad. Estos son aspectos centrales para pensar los procesos de reforma, resistencia y empoderamiento frente a los procesos opresivos, desubjetivantes que intentan arrasar subjetividades y memorias encarnadas.
\end{abstract}

\section{Palabras Clave}

salud mental comunitaria, formación profesional en salud, interdisciplina

\begin{abstract}
This article characterizes the epistemological assumptions in the training proposals of the Psychopathology Service of the Aráoz Alfaro Hospital ("the Lanús") and the postgraduate courses in Community Mental Health of the National University of Lanús (SMC UNLa). With qualitative methodologies and triangulation of sources from a comprensivist paradigm, we conducted 6 interviews with referents, 74 surveys of students and graduates of the postgraduate courses; and we surveyed 92 documents. The results highlight the implications of the Lanus in management areas, design of national policies and conformation of the Community Mental Health Area at UNLa. Both experiences with their singularities share the same epistemological basis and include formative aspects that make visible and enhance alternative historical constructions in the field of mental health as well as replicate them from the interdisciplinary and participatory experience in and with the community. These are central aspects to think reform processesf, resistance and empowerment in the face of oppressive, desubjective processes that attempt to destroy subjectivities and embodied memories.
\end{abstract}

Keywords

community mental health, health professional training, interdisciplinary

Correspondence about this article should be addressed to María Marcela Bottinelli. Email: mmbottinelli@yahoo.com.ar 


\section{LANÚS, INTERDISCIPLINARY TRAINING EXPERIENCES. BACKGROUND OF COMMUNITY MENTAL HEALTH ${ }^{2}$}

La salud mental y los problemas que la afectan han sido estudiados desde hace décadas y señalados en Informes internacionales, Consensos y Declaraciones como un campo problemático prioritario en el ámbito internacional y nacional. Los problemas de salud mental se encuentran entre las primeras causas de morbilidad y se estima que la brecha de tratamientos es aún muy profunda y con grandes desigualdades regionales (Kestel, 2018). Este preocupante cuadro de situación, encuentra en dos experiencias emergentes en Lanús aportes disruptivos a la lógica manicomial que se enmarcan en los procesos de reforma sostenidos y profundizados, con algunas interrupciones, desde hace más de 60 años.

Los análisis internacionales muestran la necesidad de focalizar en los procesos formativos que permitan, promuevan y sostengan los procesos de transformación de los paradigmas de salud mental. Las transformaciones paradigmáticas son muy difíciles de sostener y los procesos de transformaciones en el campo tensionan con intereses, prejuicios y representaciones sociales contradictorias que obstaculizan la efectivización de dichas transformaciones. La Psicología Social Comunitaria se encarga de revisar dichas modificaciones a través de las investigaciones sobre representaciones sociales, sobre identidades, sobre transformaciones $\mathrm{o}$ procesos sociales desde diferentes abordajes y conceptualizaciones tratando de comprender y desentramar dichos procesos. Esta perspectiva implica reconocer en los actores, las acciones y los discursos, las tensiones de los viejos paradigmas centrados en el sistema tutelar, el encierro y la peligrosidad, y los nuevos centrados en la protección de derechos y restitución de aquellos que fueron vulnerados, junto a las dimensiones de la participación social, el control de recursos y del poder, el fortalecimiento político de ciudadanía, la autogestión y el compromiso ético, como estructurantes de procesos historizantes y transformadores (Bottinelli, 2014).

Las experiencias que abordamos son referenciadas en diferentes espacios y estudios como formadoras y transformadoras, a la vez que han originado diferentes formas de replicación en diversos espacios en el país y la región. Estas experiencias muestran posibilidades de transformación en contextos específicos desde la potencialidad.

Lanús es uno de los 135 partidos de la provincia argentina de Buenos Aires. Forma parte del aglomerado urbano conocido como Gran Buenos Aires, con una población de 10.077,78 hab/km², es el partido más densamente poblado después de la Ciudad Autónoma de Buenos Aires en Argentina.

Las experiencias en las que nos centramos son, por una parte, la del Servicio de Psicopatología del Hospital Policlínico de Lanús “Aráoz Alfaro" (1957) dirigido por el Dr. Mauricio Goldenberg entre 1957 y 1971 y luego por Valentín Baremblit hasta 1976, conocida como "El Lanús" o "la experiencia de El Lanús". Esta experiencia es reconocida como constituyente de la creación del campo de la salud mental en Argentina (Carpintero y Vainer, 2004) y fue laboratorio de políticas públicas que tuvieron expresión concreta por ejemplo en la Ciudad de Buenos Aires (Plan Goldenberg de 1968) y a nivel nacional en el documento "Lineamientos para un Plan Nacional de Salud Mental" en el marco del retorno de la democracia en 1984. La otra experiencia es la de la creación de los posgrados en Salud Mental Comunitaria de la Universidad Nacional de Lanús, fundados y dirigidos por el Dr. Emiliano Galende. Estos emergen casi desde la misma fundación de la universidad, en 1996 y se proponen explícitamente dar continuidad en el ámbito académico al impulso de la experiencia territorial del Lanús en el ámbito de la Salud Mental Comunitaria.

Ambas experiencias son reconocidas y señaladas por diversos investigadores y referentes como fundantes en salud mental y han sido referenciadas en legislaciones, documentos y programas formativos tanto nacionales como internacionales.

La experiencia de "El Lanús" ha encontrado en el Departamento de Salud Comunitaria de la Universidad Nacional de Lanús, una línea de formación continua que se sostiene en los principales ejes de los procesos de transformación del campo de la Salud Mental, a la vez que contextúan la formación

\footnotetext{
${ }^{2}$ El presente artículo está elaborado en base al informe final de investigación del proyecto Amílcar Herrera 33A201 de la UNLa.
} 
en las crecientes necesidades de los procesos de salud enfermedad atención y cuidado, y de las Políticas Públicas actuales en el área.

Dada la coexistencia territorial, la distancia temporal, la vigencia de los valores y fundamentos de ambas experiencias, y nuestra posibilidad de acercamiento a los documentos y registros históricos, así como a algunos de los participantes y referentes de ambas experiencias, nos propusimos como objetivos caracterizar los supuestos que subyacen y expresan continuidades y rupturas respecto de actores, espacios de trabajo y formación, dispositivos, conceptos/autores y contextos de implementación; así como también indagar aspectos facilitadores, obstaculizadores y reproductores (rizomáticos) en y de las formaciones en las propuestas formativas del Servicio de Psicopatología del Htal. Aráoz Alfaro y de los posgrados en Salud Mental Comunitaria (SMC) de la UNLa.

\section{Metodologia}

La pregunta central que guió esta investigación fue: ¿Cuáles son los sustentos epistemológicos que subyacen en estas propuestas formativas fundantes y les dan continuidades y rupturas a los procesos formativos?

Empleamos un diseño predominantemente cualitativo con triangulación metodológica de fuentes desde un paradigma comprensivista con un enfoque hermenéutico-dialéctico (Minayo, 2009). El diseño fue flexible, a medida que avanzó la investigación se fueron analizando los datos recogidos, así como definiendo, reformulando las hipótesis y ajustándose los instrumentos de indagación. Las muestras fueron de tipo intencional (Rubio y Varas, 1999; Cordova, 1990) y teórica (Glasser y Strauss, 1967), seleccionando los casos de acuerdo con los objetivos del proyecto y siguiendo los criterios de heterogeneidad y accesibilidad (Valles, 2003) con corte por saturación (Minayo, 2009; Valles, 2003).

En este sentido, para el abordaje de las experiencias formativas fundantes (Lanús 1957 y posgrados UNLa), el procedimiento para la selección de las unidades de análisis implicó un método de bola de nieve (Valles, 2003) a partir de la consulta con informantes calificados y claves, que garantizaran un clima de confianza y reconocimiento en los encuentros (Nabergoi, 2013). Se incluyeron profesionales/trabajadores de la salud que participaron en las experiencias estudiadas y que tuvieron actuación en el campo de la salud mental desde distintos roles. Se realizaron entrevistas en profundidad con la modalidad de relatos de vida (Kornblit, 2007) o historias de vida tópicas (Minayo, 2009), propios del enfoque biográfico con miembros del equipo fundante del Servicio y de los posgrados (Octavio Fernández Mouján, Lía Ricón, Vicente Galli, Carlos Sluzki, Isaac Levav, Daniel Rodriguez) y se revisaron materiales videofilmados de Emiliano Galende, Valentín Baremblit, Mauricio Goldenberg, entre otros. En todos los casos se se solicitó el consentimiento informado con fines de investigación y realización audiovisual, se incluyó además una autorización específica para el uso de los nombres de los participantes en el análisis y presentación de resultados.

También se incorporaron registros escritos y videograbados de entrevistas y conferencias de protagonistas.

En el caso de participantes de los posgrados, se realizaron encuestas a estudiantes y graduados de la Maestría y el Doctorado en Salud Mental Comunitaria de la UNLa, indagando sus inserciones actuales y la relación entre su participación en dichos posgrados como experiencia formativa y sus prácticas profesionales. Se sistematizaron y procesaron las respuestas de los cuestionarios y se analizaron en función de los objetivos del proyecto

El trabajo con fuentes secundarias comprendió la revisión de Revistas, Actas de congresos y jornadas, bibliotecas y archivos institucionales que documentaban la experiencia del Servicio de Psicopatología del Htal. Aráoz Alfaro. Respecto de los posgrados de la UNLa se revisaron planes de estudio originales, presentación y resoluciones de creación de las carreras, archivo audiovisual de la Biblioteca R. Puiggrós (UNLa) y convenios para el establecimiento de redes y espacios de prácticas. Se sistematizó y analizó un corpus de 92 documentos textuales y registros audiovisuales (fuentes secundarias), que recogen la experiencia formativa de "El Lanús" (como lo denominan comúnmente quiénes han participado y/o conocido la experiencia de 1957). Dichos documentos fueron digitalizados, 
alojados en un sitio web (para el acceso exclusivo del equipo del equipo de investigación) y organizados de acuerdo al tipo de archivo y fecha de publicación y/o edición. Los materiales audiofilmados recuperados fueron sistematizados y analizados a través de una matriz de relevamiento que incluía los ejes de análisis centrales del proyecto y los emergentes. También se revisaron los documentos referidos a las carreras y la universidad como resoluciones de creación del área de salud mental comunitaria y el Centro de Salud Mental Mauricio Goldenberg, los planes de estudio de los posgrados, las resoluciones que les dieron origen así como sus modificaciones y fundamentaciones, informes de las carreras y documentos de autoevaluación y evaluación propios de la universidad y de las evaluaciones realizadas por la Comisión Nacional de Evaluación y Acreditación Universitaria (CONEAU).

\section{Resultados}

Respecto a las continuidades y similitudes entre ambas propuestas formativas destacamos que a pesar de momentos históricos y políticos diferentes encontramos que cada una de las experiencias presenta sus singularidades pero expresan un punto de vista común, una misma base epistemológica. Probablemente la proximidad territorial y las condiciones de posibilidades institucionales que se brindaron desde la UNLa fortalecieron el pasaje y expansión originarios.

La continuidad epistemológica encuentra en Lanús aportes disruptivos a la lógica manicomial, que se enmarcan en los procesos de reforma que desde hace más de 60 años fueron desarrollados en la experiencia del Servicio de Psicopatología en el Hospital Policlínico de Lanús Araoz Alfaro y, en el ámbito académico, por las propuestas formativas y acciones realizadas por el Departamento de Salud Comunitaria de la Universidad Nacional de Lanús

De los presupuestos originales destacamos aquellas prácticas transformadoras de la realidad organizacional de la época que a su vez resultaron en experiencias acumulativas que expandieron y difundieron la necesidad de cambio:

- Amplitud de criterios formativos que permitió determinar la complejidad de la práctica en salud mental al superar las limitaciones que proporciona un solo enfoque disciplinar. De este modo se han legitimado relaciones interdisciplinares amplias - en las cuales confluirían una psiquiatría más dinámica, un psicoanálisis adecuado para la atención en el hospital público, apertura al enfoque comunicacional y al enfoque sistémico, participación activa de las ciencias sociales- permitiendo coexistencias entre diferentes líneas de trabajo.

- Énfasis en la capacitación de recursos humanos especializados capaces de llevar adelante prácticas pertinentes para modificar estructuras existentes.

- Fortalecimiento del diálogo entre Salud Mental y el resto de las prácticas de salud hospitalarias. El esfuerzo de Goldenberg fue limitar la separación y estigmatización del área de Salud Mental. Las patrullas interdisciplinarias conformaron a nivel intrahospitalario el espacio de demanda e interconsulta favorecedor del acercamiento. Dichas patrullas consistían en rondas periódicas que realizaban lxs profesionales del Servicio de Psicopatología por todas las áreas del hospital, revisando las necesidades y posibles intervenciones que podían articular con los demás servicios.

Del mismo modo el pasaje de profesionales por ambos dispositivos ha marcado una impronta singular que se reproduciría a otros espacios político-institucionales en las décadas siguientes y hasta la actualidad. Por ejemplo, la influencia en quienes llevaron a cabo políticas públicas, en coordinación de programas, en investigaciones o propuestas formativas. Su expansión se ha volcado hacia la formación y asistencia en la Ciudad de Buenos Aires - con el Plan Goldenberg a fin de los años 60`s-. Del mismo destacamos que Vicente Galli, desde la Dirección Nacional de Salud Mental, reproduciría, como modelo de gestión, el formato original promovido por Goldenberg.

La expansión del Lanús se vio reflejada en multiplicidad de acciones destacando: la capacitación en acciones preventivas, creación de un Centro Nacional de Capacitación y Acreditación de nivel terciario y cuaternario coordinado junto con organismos universitarios y centros regionales de formación, 
habilitación de centros periféricos de salud mental, que contaron con hospitales de día los cuales se ejecutaron en la comunidad e instituciones representativas- escuelas, fabricas, iglesias, policía y sociedades de fomento privilegiando la atención primaria. La promoción de los derechos se vio reflejada en garantizar la accesibilidad para la atención, fomento de educación sanitaria, desarrollo de programas específicos según grupos etarios y problemas concretos- alcoholismo, retardo mental, psicosis infantiles, etc.

De modo análogo, el Área de Salud Mental Comunitaria de la UNLa tomó y expandió dichos presupuestos originales proponiendo una multiplicidad de aportes curriculares formativos que han enriquecido al campo de la salud mental comunitaria: promoción de procesos de reforma, erradicación del sistema manicomial, protección de derechos ciudadanos, caracterización desde la salud y no desde la enfermedad, cuestionamiento a la medicalización, desarrollo de prácticas interdisciplinarias e integrales de salud.

Respecto a las diferencias, la experiencia Goldenberg no plantea una estrategia confrontativa con el manicomio sino que operan evitando la derivación. De este modo se reducirían las camas y se limitaría su capacidad operativa. La hipótesis de la época era que reduciendo su capacidad operativa se iría limitando sus funciones y de este modo desaparecería porque sus funciones serían reemplazadas paulatinamente por una red de servicios. Los asilos deberían ser remplazados por Hospitales Psiquiátricos para psicosis aguda de corto período de internación.

La mirada de la experiencia del Lanús aun cuando desarrolla prácticas comunitarias en el Área Programática del Aráoz Alfaro sigue teniendo un punto de vista hospitalocéntrico. La acción médica termina siendo totalizadora por cuanto el Hospital inscribe acciones integrales promocionando la asistencia temprana, facilitando la accesibilidad y penetración en la comunidad. Como experiencia inédita para la salud pública argentina se destaca la habilitación de camas en un hospital general. Más adelante la marca de Alma Ata 20 años, la Declaración de Caracas y los diferentes programas de Reforma en la Región, suministran claridad a las renovadas propuestas formativas que se promueven desde la UNLa en cuanto a que la estrategia de Atención Primaria de la Salud (APS) permite dar visibilidad a la organización de la red de servicios, a la participación social, al desalojo del Hospital Psiquiátrico como núcleo central de la atención y a la promoción de derechos.

A diferencia del planteo formativo de la UNLa -desde la cual se promueve la atención integral y comunitaria de la salud mental- los presupuestos que fijaría el Primer Plan Nacional de Salud Mental con la consultoría de Mauricio Goldenberg como alternativas a la asistencia manicomial, resultan en la expansión de acciones de salud mental en los hospitales generales, la promoción de unidades psiquiátricas en los hospitales de mayor complejidad con una proporción de camas y consultorios en relación a la capacidad y recursos totales de los establecimientos.

Con respecto a una hipótesis que teníamos al inicio del trabajo de que hubo estrategias didácticas y dispositivos asistenciales y de formación que encarnaban las teorías y conceptos que sostienen las propuestas, encontramos que las estrategias didácticas (ateneos, grupos de estudio, reuniones de staff y por departamentos, admisiones y sesiones compartidas entre profesionales y sistemas completos de formación como las residencias) y dispositivos asistenciales (modalidad de hospital de día en el sector de internación, consultorios externos) del servicio de Lanús, encarnan conceptos que no necesariamente son explicitados en esta experiencia, pero que son retomados y desarrollados por los posgrados SMCUNLa. Aunque en estos últimos, las estrategias didácticas fomentan el trabajo interdisciplinario y articulación con las prácticas de los estudiantes en los dispositivos asistenciales en los que trabajan que devienen en contextos de implementación. También puede verse un énfasis en otros de los valores y principios UNLa que se articula con el de los equipos interdisciplinarios y la articulación teoría-práctica, que es el trabajo desde una perspectiva pedagógica de aula-taller, presentes en el Proyecto Institucional y los "Principios y Valores de la UNLa".

Respecto de la continuidad de actores, teorías, autores y determinantes contextuales históricopolíticos, encontramos que la continuidad de actores se expresa en la presencia de docentes de posgrado 
y directivos de la universidad (que habilitaron la creación de los posgrados), que participaron de distintas maneras en la experiencia del Lanús. Algunos fueron líderes como Valentín Barenblit que estuvo a cargo como Jefe del Servicio tras la partida de Goldenberg en 1971, otros fueron claves en el trabajo comunitario como Elena de la Aldea, y otros residentes como Daniel Rodríguez. También se puede observar continuidad de referentes como Isaac Levav en temas de resiliencia comunitaria; y de centralidad de temas de trabajo articulados con diferentes miembros e instituciones participantes de la Red Maristán como promoción de salud, movimientos de transformación del campo de la salud mental, participación comunitaria.

Respecto de los autores, si bien en principio pareciera no haber una continuidad estricta y completa, ésta se expresa en los posgrados en el reconocimiento a autores que a la vez son docentes y partícipes de la experiencia del Lanús. En algunos casos se retoman autores paradigmáticos de la experiencia del Lanús, tales como Pichón Riviere o el mismo Goldenberg, aunque en los programas de las materias de los posgrados SMC-UNLa no ocupan un lugar central ni son referenciados por los estudiantes como especialmente trascendentes en su formación. Sin embargo, los estudiantes y graduados señalan como referentes a quienes fueron sus docentes como Barenblit, Galende y Elena de la Aldea, quienes retoman los conceptos y autores de la experiencia del Lanús en sus clases.

Encontramos, además, una continuidad en las teorías que se expresan en la experiencia del Lanús a través de referentes de diversas corrientes teóricas como psicoanálisis, terapias sistémicas, perspectiva comunitaria, que se inscriben en la propuesta de trabajo en Salud Mental ${ }^{3}$. Por su parte, los posgrados SMC-UNLa, retoman esa tradición de la Salud Mental y las diferentes experiencias de reforma psiquiátrica y amplían a la perspectiva de derechos.

Respecto de los determinantes contextuales históricos-políticos que permitieron que las propuestas se afianzaran, si bien no se profundizó en las entrevistas este aspecto, sí aparece frecuentemente el contexto democrático, de ampliación de derechos y salud pública como facilitadores de la creación de este tipo de espacios y los procesos de dictadura como persecutorios de quienes formaban parte de estas experiencias. El retorno de la democracia y los contextos de posibilidad de creación de espacios que recuperen esas perspectivas de la salud comunitaria y del compromiso de la formación de profesionales para la solución de problemas contextuados, permiten establecer una línea de continuidad recuperando la propuesta de "El Lanús" a partir de actores y principios de aquella experiencia, reeditados y contextuados en la formación de los posgrados de SMC-UNLa.

Por otra parte, se anticipa que la territorialidad marcó un cierto hito de continuidad. Si bien la propuesta de continuidad territorial no fue referenciada explícitamente en los entrevistados, los documentos señalan claramente la preocupación por el trabajo inmerso en el contexto de los problemas concretos locales y regionales en los que se enmarcan ambas propuestas. Asimismo permiten también mostrar que los principios y los actores que los encarnan sí sostienen como un valor importante la responsabilidad de dichos espacios en la construcción de propuestas comprometidas con la comunidad en el territorio.

Por último, tanto en las experiencias relatadas en las entrevistas, como en los dichos de los estudiantes en las encuestas, el paso por ambos espacios de formación (más allá de la titulación), ha transformado sus perspectivas y marcado su perfil profesional como identitario. Generando espacios tanto en la clínica como en la formación, como en diferentes niveles de la gestión y la investigación, que promueven abordajes complejos, interdisciplinarios, de carácter comunitario, con énfasis en la resolución de los problemas con perspectiva de derechos.

\footnotetext{
${ }^{3}$ Dichos referentes son quienes migraron en los procesos de dictadura hacia otros países, desde los cuáles contribuyeron al campo no solo desde la revisión teórica y la articulación entre sus formaciones de base con la Psicología Social Comunitaria, sino replicando los modelos de trabajo aprendidos en El Lanús. (Ej. Carlos Sluzki -Escuela Sistémica en Palo Alto con intercambios con desarrollos desde el psicoanálisis con perspectiva dialéctica en México-, Mauricio Goldenberg -quien constribuyó al desarrollo de un Plan Nacional de Salud Mental durante su exilio en Venezuela-, Itzaak Levav -con influencia en SMC a nivel regional e internacional a través de OPS-OMS-, Valentín Barenblit -primero en Venezuela y luego desarrollo de psicoanálisis en Barcelona a través del centro IPSI-, Elena de la Aldea, entre otrxs).
} 
Así explican los referentes de ambas propuestas dichos espacios de formación:

Goldenberg desarrolla la noción de ideología del servicio, que se relaciona con un enfoque que denominaban "enfoque psicosomático-social". Se incluye en la descripción de esta "ideología" la

inquietud por lograr una completa integración de la Psiquiatría en el Hospital General expresando la preocupación por unir los aspectos médicos y psicológicos en la comprensión del hombre enfermo como totalidad (...) [en el] campo de la docencia y en el de la investigación, unimos los modernos aportes de la psiquiatría dinámica -y de la psicología social con los estudios de la psiquiatría clínica. La modificación de esquemas referenciales tradicionales exige actitud plástica, pero crítica, para que ésta se realice en forma coherente. (...) [El] desarrollo grupal se facilita por una relación interpersonal no discriminatoria por prejuicios raciales, políticos, religiosos, etc., y por la tolerancia y respeto hacia las distintas orientaciones teóricas individuales, permitiendo una coexistencia doctrinaria, el intercambio y la colaboración. Lo característico de nuestra actitud es el intento de superar las limitaciones de un mero ejercicio técnico en un esfuerzo permanente por comprender, y comprometerse, con la totalidad de la condición humana. Esto se traduce en una tarea científica, asistencial, preventiva y social. El compromiso se da en la labor común institucionalizada. No queremos participar de una psiquiatría universal abstracta, injertada en nuestro país. Sensibles a los problemas nacionales pretendemos impulsar una psiquiatría nuestra, atenta a ciertas características peculiares del hombre argentino. Esto no significa propugnar una actitud aislacionista, sino por el contrario, realizar en nuestro medio los postulados que sustenta la Organización Mundial de la Salud. (...) En síntesis, creemos que una ideología se elabora y se concreta en el trabajo, de lo contrario constituye un intelectualismo desarraigado o un activismo vacío. De esta manera el trabajo se hace más rico y apasionante. Intentamos que cada integrante del grupo se sienta parte de algo que hay que realizar además de llevar adelante lo realizado. Estar en el Servicio es un compromiso, además de profesional, humano; en él se lucha por la asistencia específica de "lo enfermo" y el mejoramiento del hombre sano y de su ámbito (Goldenberg y cols., 1966, págs. 82-83).

Por su parte, Galende, al presentar el Centro de Salud Mental Comunitario "Mauricio Goldenberg" de la UNLa, describe

Desde el punto de vista epistemológico, el área de la salud mental comunitaria es un nuevo campo teórico en transición, que avanza desde su origen en la medicina hasta su conformación actual como disciplina social.

Su carácter multidisciplinario, la articulación de conocimientos y prácticas de las ciencias sociales y de la salud, hace que requiera de un debate permanente a fin de permitir una incorporación crítica de conceptos, categorías y valores de estas ciencias al nuevo campo teórico que constituye la salud mental.

La dimensión social de la salud mental es concordante con las prioridades fijadas por la Universidad Nacional de Lanús: el sostenimiento de una universidad comprometida con las condiciones de la vida social de su comunidad. Estos tres ejes, el de la producción de conocimientos, el del diseño de nuevas prácticas en salud y el ejercicio práctico del compromiso de la Universidad con la sociedad y sus necesidades, conforman un mismo y simultáneo objetivo del área. 
La sanción de la Ley Nacional de Salud Mental y su Decreto Reglamentario enmarcan la responsabilidad universitaria de revisar las prácticas profesionales instituidas y formar profesionales que puedan desarrollar acciones acordes a un Modelo Comunitario de Atención sin instituciones monovalentes. Este Modelo implica sostener una perspectiva de Derechos Humanos para el trabajo con las personas con padecimiento psíquico y sus familias.

En este marco resulta fundamental la existencia de una Universidad comprometida con las condiciones de la vida social de su comunidad, y su colaboración con las políticas públicas en este sector.

La producción de conocimientos, el diseño de nuevas prácticas en salud y el ejercicio concreto del compromiso de la universidad con la sociedad y sus necesidades, conformaron un mismo y simultáneo objetivo del programa de trabajo del área (Galende, $2011, \mathrm{~s} / \mathrm{p})$.

\section{Conclusiones}

La "densidad" de los relatos, la intensidad de las experiencias y sus actores, y el cruce de textos y voces generó una riqueza y complejidad en la información recolectada que implicó mayor tiempo de análisis, interpretación y reinterpretación de resultados que debieron acotarse para esta presentación. Seguimos trabajando con ellos en vistas a profundizar las lecturas que puedan mostrar los diferentes niveles de análisis y resultados obtenidos, y redundar en productos que promuevan una revisión de la historia y una prospectiva de los alcances de estas experiencias en el campo actual de la salud mental comunitaria, en el marco de los procesos de transformación de salud mental promovidos por la Ley Nacional de Salud Mental N 26657 y de los festejos de los 20 años de los posgrados de Salud Mental Comunitaria de la UNLa.

La mirada que sostiene este trabajo, con base en diversos aportes de la Psicología Social Comunitaria y desde la cual recuperamos estas experiencias, se enmarca entonces

desde una perspectiva que, sin desconocer los aspectos subjetivos del aprendizaje, requiere integrar la historia y el contexto como determinantes de los significados que adquieren los procesos educativos en el campo de la promoción y educación para la salud mental, situados en la compleja red de relaciones y tensiones - de poder y saber entramadas entre los diferentes actores sociales que participan en la vida cotidiana en los procesos de salud enfermedad atención y cuidado. (Bottinelli, 2014, pag 329)

El actual contexto sociopolítico y el proceso de implementación de la Ley Nacional de Salud Mental nos interpela a recuperar y preservar las experiencias formativas innovadoras en el campo de la Salud Mental Comunitaria, y vuelve a poner en juego esas continuidades y rupturas en las tensiones y procesos de lucha por derechos en el campo de salud mental. En este marco, analizar dichas propuestas, así como recuperar las experiencias comunitarias, los saberes y prácticas construidos en contextos contradictorios, históricamente alternativos y opuestos a las hegemonías dominantes, implica visibilizar esos procesos y voces de los actores para conocer, compartir y recuperar esas luchas sin idealizarlas, pudiendo comprender en el presente tanto la reedición de estas tensiones como las estrategias de resistencia construidas colectivamente. Entre ellas las propuestas estudiadas incluyen aspectos formativos que permiten visibilizar y potenciar esas construcciones así como replicarlas desde la experiencia interdisciplinaria y participativa en y con la comunidad, aspectos centrales para pensar los procesos de reforma, resistencia y empoderamiento frente a los procesos opresivos, desubjetivantes que intentan arrasar subjetividades y memorias encarnadas. 


\section{Referencias}

Bottinelli, M.M. (2014). Promoción y educación para la salud en los planes y políticas de Salud Mental en Argentina. Dir: Emiliano Galende. Tesis de doctorado en salud mental comunitaria. UNLa.

Carpintero, E. \& Vainer, A. (2004) Las huellas de la memoria. Psicoanálisis y Salud Mental en la Argentina de los '60 y '70. Tomo I: 1957 - 1969. Buenos Aires: Topía

Córdova, V. (1990). Historias de vida. Una metodología alternativa para Ciencias Sociales. Caracas: Fondo editorial Tropykos. Comisión de Estudios de Posgrado. FACES/U.C.V.

Galende, E. (2011) Centro de Salud Mental Comunitaria "Dr. Mauricio Goldenberg" Presentación. Disponible en http://www.unla.edu.ar/index.php/centro-de-salud-mental-comunitaria-presentacion

Glasser, B. \& Strauss, A. (1967). El descubrimiento de la teoría emergente. Traducción de un capítulo del libro Strategy for qualitative reserch. New York: Ed. Aldine. Mimeo.

Goldenberg, M.; Barenblit, V.; Fernandez Moujan, O.; Galli, V.A.; Kesselman, H. Muller, A.; Perez, A.; Ricón, G.L.; Sluzki, C.E. \& Stein, G. (4 de enero de 1966). La Psiquiatría en el Hospital General. Historia y estructura del Servicio de Psicopatología y Neurología del Policlínico "Profesor Dr. Gregorio Araoz Alfaro". En sección Psiquiatría de la revista Semana Médica Nº1.

Kestel, D. (2018) Presentación sobre la Salud Mental en las Américas. OPS-OMS

Kornblit, A.L. (2007). Metodologías cualitativas: modelos y procedimientos de análisis. Buenos Aires: Biblios.

Minayo, M.C. Souza (2009). La artesanía de la investigación cualitativa. Buenos Aires: Lugar Editorial.

Nabergoi, M. (2013) El proceso de transformación de la atención psiquiátrica hacia el enfoque de cuidados en salud mental en Argentina. Participación de Terapia Ocupacional en la construcción del campo de la salud mental en la Ciudad de Buenos Aires (1957-1976). Tesis de Doctorado en Salud Mental Comunitaria. UNLa.

Rubio, J. \& Varas, J. (1999). El análisis de la realidad, en la intervención social. Métodos y técnicas de Investigación. Madrid: Ed. CCS.

Valles, M.S. (2003). Técnicas cualitativas de investigación social. Reflexión metodológica y práctica profesional. Madrid: Ed. Síntesis. 